\title{
Vital Nodes Evolution Study on Railway Network of Silk Road Economic Belt
}

\author{
Yanbo Zhu1, Hening Fan² \\ ${ }^{1}$ School of Physics \& Electronic Information Engineering, Qinghai Nationalities University, Xining, China \\ ${ }^{2}$ School of Mathematics, Qinghai Nationalities University, Xining, China \\ Email: ybzhu6616@163.com
}

Received 13 February 2016; accepted 6 August 2016; published 9 August 2016

Copyright (C) 2016 by authors and Scientific Research Publishing Inc.

This work is licensed under the Creative Commons Attribution International License (CC BY).

http://creativecommons.org/licenses/by/4.0/

c) (i) Open Access

\begin{abstract}
In order to study the nodes importance and its evolution process of the railway network of SREB (Silk Road Economic Belt), we construct the network (RNSREB) based on Graph Theory, which focuses on the time intervals according to actually railway network, railway project under construction and the national railway network of medium-and long-term plan. The algorithms for vital nodes evaluation are analyzed, the evaluation method on nodes importance of RNSREB is proposed, the quantized values of each node are calculated with Pajek, and TOP20 core nodes of the network with different coefficients and time intervals are determined respectively. Then the evolution process of TOP20 critical nodes with 4 periods is contrasted and analyzed. It is indicated that some vital nodes newly discovered (Geermu, Maduo, Ruoqiang) should be concerned.
\end{abstract}

\section{Keywords}

Graph Theory, Node, Complex Network, Silk Road Economic Belt (SREB), Railway Network of Silk Road Economic Belt (RNSREB)

\section{Introduction}

Silk Road connects Europe civilization, East Asia civilization and Islam civilization, and also builds the exchange bridge for culture and economy of West and East, which has the history for more than two thousand years. In order to exploit domestic advantage and improve the developing level of different area, Chinese Government proposed the strategy of the Silk Road Economic Belt (SREB) and the 21st-Century Maritime Silk Road, the Belt and the Road is a national strategy and has important sense for China's modernization and leader's position of the global. The B \& R will also tackle the conundrum of weak strategic channel of China, and promote development of trade and economy with Mid Asia, West Asia, South Asia, Europe and Africa [1]. 
The northwest and southwest areas of China locate in the dominate position of the Belt; strengthening the exchange and cooperation with Mid Asia, West Asia, South Asia and European countries is also part of national strategy. After construction for many years, the railway network of these areas has been built and plays a very important role [2] [3]. With the lines of Lanzhou-Chengdu (Chongqing), Xian-Chengdu, Chongqing-Kunming, Geermu-Kuerle, Dunhuang-Geermu, Hetian-Ruoqiang, Chengdu-Geermu, Chengdu-Xining being built, the railway will play a more important role.

Graph Theory is widely used in various complex traffic networks as aviation network, bus network, highway, railway network, etc. Some scholars have studied different traffic networks like highway network, air line network, city bus line and stop network with it, and get very good result.

Wang evaluated the evolution process of China's air transport network (ATNC) during 1952-2008 with graph index and complex network methods, and explored the allometric growth in the development history of ATNC with the fluctuating growth of nodes (cities) and edges (airlines or city pairs), of which the average path length in ATNC reduced from 5.74 in 1952 to 2.24 in 2008, and the clustering coefficient rose from 0 to 0.69 [4]. Wu explored the bus network of Beijing from two aspects, found that the network has the character of typical complex network, and analyzed the character of validation and anti-attack of it with random and intentional attack [5].

Wang proposed the method for core nodes and edges identification of railway network, which need to consider the required amount of transportation and prior level of cargo transportation; they also denoted the plan of repair sequence under emergency [6]. Chu constructed Wuhan Bus Stop Network; they studied the survivability of the network with two policies of random and intentional attack, and measured it with average shortest path, relative size of biggest connected sub-graph and average clustering coefficient [7].

This paper analyzes the RNSREB (Domestic Part) with complex network and graph theory, studies the algorithms on how to determine the core nodes (city or traffic hub), provides an algorithm to identify these nodes, and calculates the quantitative value of nodes importance with adjustable coefficient at different time intervals. The contrastive analyses on the importance of the evolution process of these key nodes are listed; some proposals are given for logistics, transportation, planning and related decision support departments. Many references above only considered various static networks or benchmark networks, while this article studies the evolution process of RNSREB and each node from a dynamic view.

\section{Construction of RNSREB}

Graph theory is widely used in various complex networks such as communication network, social and economic network, logistic network, sensor network, Internet and so on. A realistic transportation network may be abstracted as a graph formed by nodes set $\mathrm{V}$ and edges set $\mathrm{E}$, each node in $\mathrm{V}$ represents a traffic hub, such as a railway station, an airport, a city, or a bus stop etc. Each edge in E denotes the line that connect two airports, two railway station, two cities or two bus station between two nodes. So the problem of transportation network may be converted to graph issue.

After integrated analysis with various kinds of railway and time intervals within SREB, such as high-speed rail, double line, single line and meter gauge railway, the RNSREB are constructed as Figure 1 and Figure 2 with Pajek. Figure 1 shows the RNSREB in Dec. 2014 after the Lanzhou-Xinjiang high-speed rail to be open. Figure 1 has 60 nodes and 75 edges. Figure 2 shows the RNSREB in Dec. 2015, including railway projects under construction, such as the railway of Geermu-Kuerle, Dunhuang-Geermu, high-speed railway of LanzhouChengdu, Chongqing-Kunming, Xian-Chengdu etc., and the projects of National railway plan like ChengduXining, Chengdu-Geermu, Hetian-Ruoqiang, Xingjiang-Xizang, Sichuan-Xizang, Yunnan-Xizang etc. Figure 2 has 70 nodes and 119 edges. The nodes in Figure 1 and Figure 2 denote cities or railway stations, the edges in it represent different railways that link these nodes. Each node may be identified by city name or Number.

From Figure 1 we can see that RNSREB is an unbalanced network, the rail density in east RNSREB is high while in the west is low. RNSREB is also a vulnerable network, any fault of node like Tulufan, Hami, Guazhou, Zhangye, Jiayuguan, Kuerle, Akesu etc. will cause the network split to two subnet. From Figure 2 we can see that the structure of RNSREB is better, especially the completion of some railway projects in middle and west of RNSREB. The importance of critical nodes in these areas will increase clearly. The doted lines in Figure 2 present the railway projects in the National railway network Medium-and Long-Term Plan and not under construction yet. RNSREB is a complex network with the character of low density, key role, diversity of compose and wide coverage area. Such character matches the reality of population and economy scale of each area in SREB. 


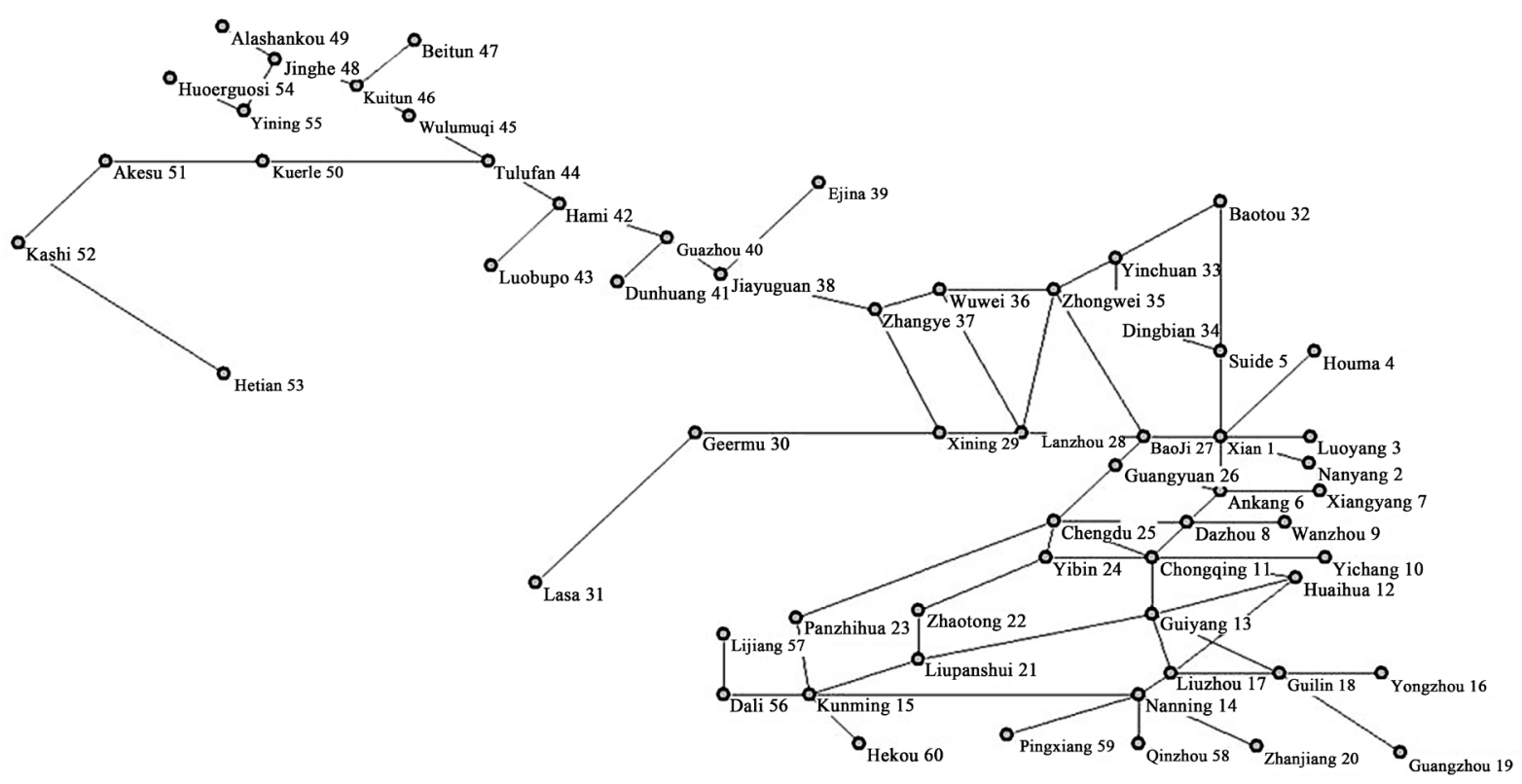

Figure 1. RNSREB (Dec., 2014, after Lanzhou-Xinjiang high-speed rail to be open).

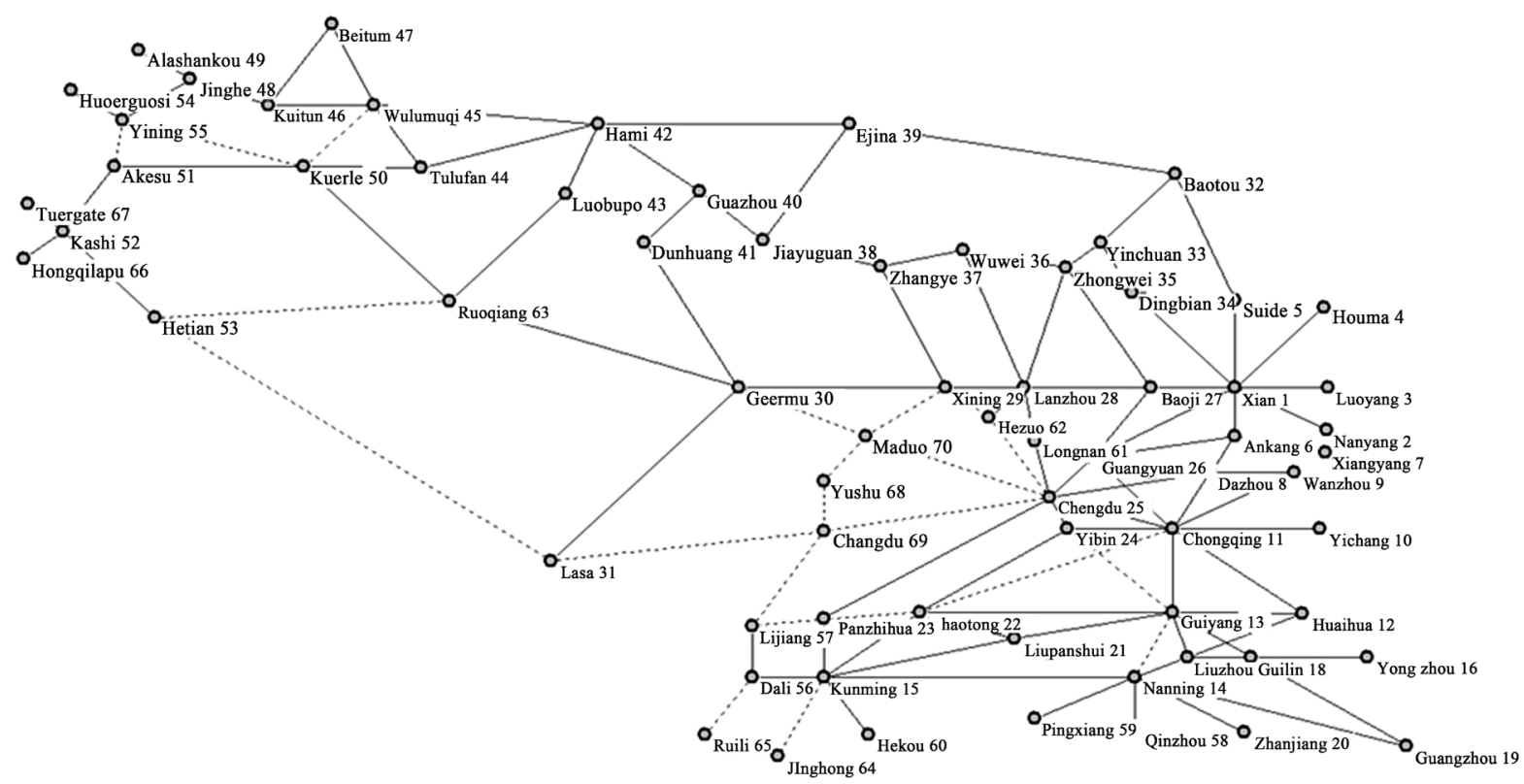

Figure 2. RNSREB (Dec., 2015, include rail project under Construction and National Railway Network Medium- and LongTerm Plan).

\section{Identification of Core Nodes}

The non-homogeneous topology structure of complex network in nature resolve that the importance of each key node is different, just like each person play different role in a department or team. The study on metrics and ranking of critical nodes importance is heating-up day by day, mining the nodes of various complex networks and focus on its characteristics analyze has important meaning. There are many methods for identifying core nodes or evaluate its importance, such as degree, eigenvector, betweenness, closeness, subgraph, node removing, node shrinking, node cluster etc, all of these are derived from Graph Theory and data mining from graph in nature.

In [8] the authors reviewed the metrics for node importance ranking in complex networks and summarized the latest progresses in this field from two prospects: the network structure and the spreading dynamics, analyzed 
the merits, weaknesses and applicable conditions of different node importance ranking metrics. Reference [9] proposed a multi-attribute decision-making method to identify the key nodes in complex networks. In their method, each node is regarded as a solution, and each importance evaluation criterion as one solution's attribute. Then, the closeness between each solution and the ideal solution were calculated in order to obtain the integration results of node importance in complex networks.

Reference [10] proposes the identification method for core nodes. It indicates that the core nodes are those that network service will be disconnected or performance decreased when it is disabled or being malicious attacked. The core nodes can be identified by node cut or edge cut algorithm. In Reference [11] the authors propose an invulnerability evaluation model based on the number of average equivalent shortest path of the whole network. Based on the model the authors also state an evaluation method for node importance, the node is more important if the network invulnerability decreased more when the node disabled.

In [12] the authors generalize the core node mining methods in several disciplines under complex network conditions, such as social network, system science, information search, literature search etc. Some important methods of it are discussed and analyzed. Reference [13] proposes an evaluation method focus on the importance of communication nodes. The relative importance of two groups of nodes in the graph can be compared with respect to the number of spanning trees. The most vital nodes are those whose removal with their incident links most drastically decreases the number of spanning trees.

In various essential study on complex networks, mining core nodes and evaluate its importance, has important practical meaning. The value of nodes degree reflect its direct influence to other nodes of the network, the closeness of nodes reflex the difficulty level reaching other nodes, which is an important index that evaluating whether the nodes in the center of the network or not, the betweenness of nodes reflect its interconnect function in the network, which is a bridge quota [14]. So we choose degree, betweenness and closeness as the index for evaluating the nodes importance of the network. As the degree value is great than 1 , while the value of closeness and betweenness is less than 1 , there is no comparative. So we defined three definitions of degree importance, betweenness importance and closeness importance to normalize these values.

Definition 1 (Degree Importance): the degree importance of node $i C_{d}^{k}(i)$ defined as the degree of node $i(d$ (i)) divide the sum of all nodes degree. The formula as follow:

$$
C_{d}^{k}(i)=d(i) / \sum_{j=1}^{m} d(j)
$$

Definition 2 (Betweenness Importance): betweenness importance of node $i C_{b}^{k}(i)$ defined as the betweenness of node $i C_{b}(i)$ divide the sum of all nodes betweenness. The formula as follow:

$$
C_{b}^{k}(i)=C_{b}(i) / \sum_{j=1}^{m} C_{b}(j)
$$

Definition 3 (Closeness Importance): closeness importance of node $i C_{c}^{k}(i)$ defined as the degree of node $i$ $C_{C}(i)$ divide the sum of all nodes closeness. The formula as follow:

$$
C_{c}^{k}(i)=C_{c}(i) / \sum_{j=1}^{m} C_{c}(j)
$$

As the single index can not reflect the node centrality effectively, here we define the nodes importance evaluating metrics mixed by degree importance, betweenness importance and closeness importance. The formula as follow:

$$
C_{H}(i)=\alpha C_{d}^{k}(i)+\beta C_{b}^{k}(i)+\gamma C_{c}^{k}(i)
$$

The coefficient of $\alpha, \beta, \gamma$ in Formula (4) satisfy the condition of $\alpha \geq 0, \beta \geq 0, \gamma \geq 0$ and $\alpha+\beta+\gamma=1$. The value of coefficient $\alpha, \beta, \gamma$ can be adjusted according to the specific characteristics of real network when we evaluating the node importance of a static network, so as to reflect the relative essentiality of evaluation indicator.

After each value of node importance $C_{H}(i)$ is calculated according to Formulas (1)-(4), we choose Top20 of $C_{H}(i)$ as vital nodes. Top20 Key nodes importance of RNSREB at different time interval is listed in Table 1. Here we take $\alpha=0.4, \beta=0.4, \gamma=0.2$ as the experience value.

The evolution list of Top20 key nodes of RNSREB after Lanzhou-Xinjiang High-speed Rail to be open is 
Table 1. Top20 key nodes importance of RNSREB at different time intervals.

\begin{tabular}{|c|c|c|c|c|c|c|c|c|}
\hline \multirow{2}{*}{ ID } & \multicolumn{2}{|c|}{ Before LanXin Rail open } & \multicolumn{2}{|c|}{ After LanXin Rail open } & \multicolumn{2}{|c|}{ Include building project } & \multicolumn{2}{|c|}{ Include future plan } \\
\hline & City & $C_{H}(i)$ & City & $C_{H}(i)$ & City & $C_{H}(i)$ & City & $C_{H}(i)$ \\
\hline 1 & Baoji & 0.0453 & Baoji & 0.0452 & Guangyuan & 0.0468 & Chengdu & 0.0573 \\
\hline 2 & Chengdu & 0.0429 & Chengdu & 0.0429 & Xi'an & 0.0440 & Chongqing & 0.0413 \\
\hline 3 & Wuwei & 0.0403 & Zhangye & 0.0391 & Chongqing & 0.0427 & Geermu & 0.0379 \\
\hline 4 & Jiayuguan & 0.0387 & Jiayuguan & 0.0385 & Lanzhou & 0.0409 & Xi'an & 0.0331 \\
\hline 5 & Guangyuan & 0.0378 & Guangyuan & 0.0379 & Chengdu & 0.0372 & Guangyuan & 0.0330 \\
\hline 6 & Zhangye & 0.0364 & Guazhou & 0.0361 & Hami & 0.0346 & Maduo & 0.0314 \\
\hline 7 & Guazhou & 0.0364 & Lanzhou & 0.0341 & Kunming & 0.0307 & Ruoqiang & 0.0311 \\
\hline 8 & Zhongwei & 0.0349 & Hami & 0.0335 & Xining & 0.0284 & Kuerle & 0.0292 \\
\hline 9 & Hami & 0.0338 & Chongqing & 0.0329 & Geermu & 0.0281 & Kunming & 0.0267 \\
\hline 10 & Lanzhou & 0.0330 & Tulufan & 0.0313 & Wulumuqi & 0.0270 & Nanning & 0.0258 \\
\hline 11 & Chongqing & 0.0323 & Wuwei & 0.0305 & Longnan & 0.0260 & Xining & 0.0212 \\
\hline 12 & Tulufan & 0.0316 & Xi'an & 0.0304 & Nanning & 0.0250 & Panzhihua & 0.0207 \\
\hline 13 & Xi'an & 0.0311 & Zhongwei & 0.0302 & Baoji & 0.0213 & Lanzhou & 0.0198 \\
\hline 14 & Kunming & 0.0300 & Kunming & 0.0293 & Ruoqiang & 0.0198 & Hami & 0.0192 \\
\hline 15 & Nanning & 0.0240 & Xining & 0.0240 & Zhongwei & 0.0189 & Wulumuqi & 0.0190 \\
\hline 16 & Ankang & 0.0232 & Nanning & 0.0233 & Kuerle & 0.0187 & Hetian & 0.0174 \\
\hline 17 & Dazhou & 0.0215 & Ankang & 0.0228 & Dazhou & 0.0166 & Zhaotong & 0.0172 \\
\hline 18 & Liuzhou & 0.0201 & Dazhou & 0.0212 & Guilin & 0.0164 & Kashi & 0.0166 \\
\hline 19 & Wulumuqi & 0.0199 & Wulumuqi & 0.0197 & Liuzhou & 0.0158 & Yining & 0.0164 \\
\hline 20 & Jinhe & 0.0169 & Guilin & 0.0181 & Ankang & 0.0157 & Yibin & 0.0157 \\
\hline
\end{tabular}

show in Table 2. The number in the column means the nodes importance ranking at different time intervals, the same as in Table 3 \& Table 4. The evolution list that includes the project under construction is show in Table 3. The evolution list of Top20 key nodes of RNSREB that include the National railway network Medium-and Long-Term Plan is show in Table 4.

$\mathrm{A}$ and $\mathrm{B}$ in Tables 2-4 means the time interval that before and after the Lanzhou-Xinjiang high-speed rail to be open. $\mathrm{C}$ indicates the time when the projects of RNSREB under construction to be open, and D present the period when the project of National Railway Network Plan to be complete and open.

\section{Key Nodes Analysis}

By the study with Figure 1, we can find that RNSREB is vulnerable, breakdown in each node like Zhangye, Jiayuguan, Guazhou, Hami or Tulufan RNSREB will split into two subnetwork, so these nodes are very important, fault in each node like Kunming, Nanning, Xian, Chongqing will cause separate node appear, so these nodes are important. While in Figure 2, RNSREB is stronger, none nodes in Table 3 \& Table 4 has such effect.

From Table 1 we can see that only Chengdu and Guangyuan ranking in the Top5 at 4 different time intervals, traffic center like Chongqing, Xian, Lanzhou, Kunming, Nanning, Wulumuqi and Hami list in the Top20 of 4 columns. Some vital nodes like Zhangye, Guazhou, Jiayuguan, Tulufan, Wuwei in the column before and after Lanzhou-Xinjiang High-speed Rail to be open disappeared in later column. The reason is that at A and B time intervals, these nodes are the only pass connect the railway from Lanzhou to Wulumuqi, so they are very important, while at $\mathrm{C}$ and $\mathrm{D}$ period, we have substitute path. 
Table 2. Top20 key nodes of RNSREB evolution list (after Lanzhou-Xinjiang high-speed rail open).

\begin{tabular}{ccccccccccccc}
\hline ID & CITY & A & B & C & D & ID & CITY & A & B & C & D \\
\hline 1 & Baoji & 1 & 1 & 13 & 24 & 11 & Wuwei & 3 & 11 & 30 & 42 \\
2 & Chengdu & 2 & 2 & 5 & 1 & 12 & Xian & 13 & 12 & 2 & 4 \\
3 & Zhangye & 7 & 3 & 26 & 30 & 13 & Zhongwei & 8 & 13 & 15 & 22 \\
4 & Jiayuguan & 4 & 4 & 28 & 36 & 14 & Kunming & 14 & 14 & 7 & 9 \\
5 & Guangyuan & 5 & 5 & 1 & 5 & 15 & Xining & 26 & 15 & 8 & 11 \\
6 & Guazhou & 6 & 6 & 33 & 37 & 16 & Nanning & 15 & 16 & 12 & 10 \\
7 & Lanzhou & 10 & 7 & 4 & 13 & 17 & Ankang & 16 & 17 & 20 & 26 \\
8 & Hami & 9 & 8 & 6 & 14 & 18 & Dazhou & 17 & 18 & 17 & 21 \\
9 & Chongqing & 11 & 9 & 3 & 2 & 19 & Wulumuqi & 19 & 19 & 10 & 15 \\
10 & Tulufan & 12 & 10 & 27 & 41 & 20 & Guilin & 32 & 20 & 18 & 25 \\
\hline
\end{tabular}

Table 3. Top20 key nodes of RNSREB evolution list (including the Project under Construction).

\begin{tabular}{ccccccccccccc}
\hline ID & CITY & A & B & C & D & ID & CITY & A & B & C & D \\
\hline 1 & Guangyuan & 5 & 5 & 1 & 5 & 11 & Longnan & & & 11 & 34 \\
2 & Xian & 13 & 12 & 2 & 4 & 12 & Nanning & 15 & 16 & 12 & 10 \\
3 & Chongqing & 11 & 9 & 3 & 2 & 13 & Baoji & 1 & 1 & 13 & 24 \\
4 & Lanzhou & 10 & 7 & 4 & 13 & 14 & Ruoqiang & & & 14 & 7 \\
5 & Chengdu & 2 & 2 & 5 & 1 & 15 & Zhongwei & 8 & 13 & 15 & 22 \\
6 & Hami & 9 & 8 & 6 & 14 & 16 & Kuerle & 23 & 25 & 16 & 8 \\
7 & Kunming & 14 & 14 & 7 & 9 & 17 & Dazhou & 17 & 18 & 17 & 21 \\
8 & Xining & 26 & 15 & 8 & 11 & 18 & Guilin & 32 & 20 & 18 & 25 \\
9 & Geermu & 30 & 32 & 9 & 3 & 19 & Liuzhou & 18 & 25 & 19 & 35 \\
10 & Wulumuqi & 19 & 19 & 10 & 15 & 20 & Ankang & 16 & 17 & 20 & 26 \\
\hline
\end{tabular}

Table 4. Top20 key nodes evolution list (including National Railway Network Medium- and Long-Term Plan).

\begin{tabular}{|c|c|c|c|c|c|c|c|c|c|c|c|}
\hline ID & CITY & A & B & $\mathrm{C}$ & $\mathrm{D}$ & ID & CITY & A & B & $\mathrm{C}$ & $\mathrm{D}$ \\
\hline 1 & Chengdu & 2 & 2 & 5 & 1 & 11 & Xining & 26 & 15 & 8 & 11 \\
\hline 2 & Chongqing & 11 & 9 & 3 & 2 & 12 & Panzhihua & 21 & 21 & 18 & 12 \\
\hline 3 & Geermu & 30 & 32 & 9 & 3 & 13 & Lanzhou & 10 & 7 & 4 & 13 \\
\hline 4 & Xian & 13 & 12 & 2 & 4 & 14 & Hami & 9 & 8 & 6 & 14 \\
\hline 5 & Guangyuan & 4 & 5 & 1 & 5 & 15 & Wulumuqi & 19 & 19 & 10 & 15 \\
\hline 6 & Maduo & & & & 6 & 16 & Hetian & 45 & 45 & 50 & 16 \\
\hline 7 & Ruoqiang & & & 14 & 7 & 17 & Zhaotong & 35 & 36 & 25 & 17 \\
\hline 8 & Kuerle & 23 & 25 & 16 & 8 & 18 & Kashi & 33 & 34 & 34 & 18 \\
\hline 9 & Kunming & 14 & 14 & 7 & 9 & 19 & Yining & 34 & 35 & 35 & 19 \\
\hline 10 & Nanning & 5 & 16 & 12 & 10 & 20 & Yibing & 24 & 26 & 29 & 20 \\
\hline
\end{tabular}


From Table 2 we can see that the ranking of Xining, Zhangye and Guilin improved a lot, Wuwei and Zhongwei decreased clearly, the others have little change from A to B. The reason is that Guiyang-Guilin-Guangzhou high-speed rail opened the same time as Lanzhou-Xinjiang rail to be open, the others has no change.

From Table 3 we can find the node Baoji which ranked No.1 in the column A and B, dropped to 13 and 24 in column $\mathrm{C}$ and $\mathrm{D}$, as it is the most important node to connect northwest and southwest of china railway before and after Lanzhou-Xinjiang High-speed Rail to be open, after the project of Lanzhou-Chengdu, Xian-Chengdu, Xining-Chengdu and Chengdu-Geermu to be open, its importance decreased obviously. With the rail projects of Lanzhou-Chengdu (Chongqing), Dunhuang-Geermu, Geermu-Kuerle, Xian-Chengdu, Nanning-Guangzhou, Yinchuan-Xian etc. to be complete and to be open, we can see that the Ranking of Xian, Xining, Wulumuqi and Kunming improved a lot, Guangyuan, Nanning and Lanzhou also rising clearly.

From Table 4 we can find the importance of Muoduo and Ruoqiang improved a lot, the importance of Geer$\mathrm{mu}$, Kuerle and Panzhihua improved obviously after the rail of Chengdu-Geermu, Chongqing-Kunming, Sichuan-Lasa, Xinjiang-Lasa, Yunnan-Lasa, Hetian-Ruoqiang etc. (the doted line in Figure 2) to be open, which are belong to National railway network Medium- and Long-Term Plan. These cities will be the critical nodes in RNSREB. The nodes ranking of Hetian, Kashi, Yining also rising a lot, Yibin and Zhaotong improved obviously.

From Tables 1-4 we can see the evolution process of Top20 critical nodes at 4 different time intervals. With more and more railway projects to be opened, some vital nodes at first became less important, while some piddling nodes ranking front.

Figure 3 shows the nodes importance at different intervals with $\alpha=0.4, \beta=0.4, \gamma=0.2$, while Figure 4 with $\alpha=0.2, \beta=0.4, \gamma=0.4$. (The value of horizontal axis in Figure $3 \&$ Figure 4 is the same as the nodes marked in Figure 1 \& Figure 2). The time intervals of A, B, C, D in Figure 3 \& Figure 4 is the same as in Tables 2-4. From Figures 3 \& Figure 4, we can see there is little difference, the importance of core nodes at different time intervals also has little difference, which validate the effectiveness of our evaluating method.

\section{Conclusions and Future Work}

This paper analyzed the nodes characteristics of RNSREB with Pajek, identified the Top20 key nodes at 4 different time intervals, and analyzed the importance evolution process of these nodes. The RNSREB (domestic part) is a complex network with the character of large scale, composing diversity and dominant status. With the completion of the rail project under construction, especially the completion of the project of Medium- and LongTerm National Railway Plan, the RNSREB will change a lot, and the robustness of it will improve sharply.

With the improvement of RNSREB structure, the importance of some nodes like Baoji, Wuwei, Zhangye, Jiayuguan, Guazhou etc. decreased, while the importance of others like Xining, Geermu, Ruoqiang, Kuerle, Maduo, Panzhihua, etc. increased. The vital nodes like Chengdu, Chongqing, Xi'an, Lanzhou, Kunming, Nanning will still play the role as dominant traffic center; there is a tendency that some new railway stations or logistics centers

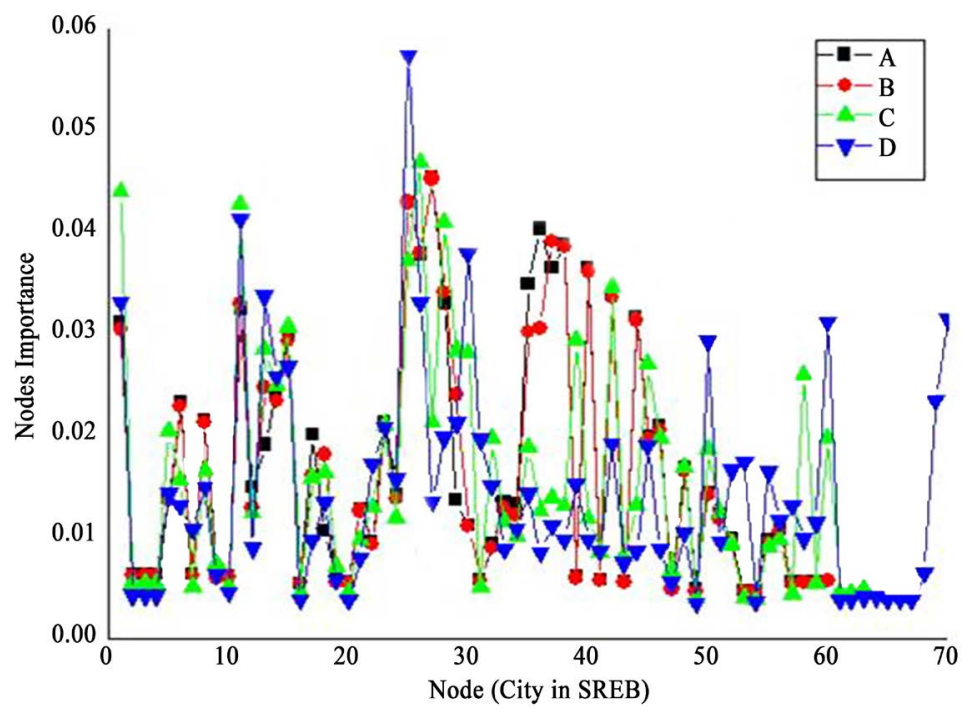

Figure 3. Nodes importance of RNSREB with $\alpha=0.4, \beta=0.4, \gamma=0.2$. 


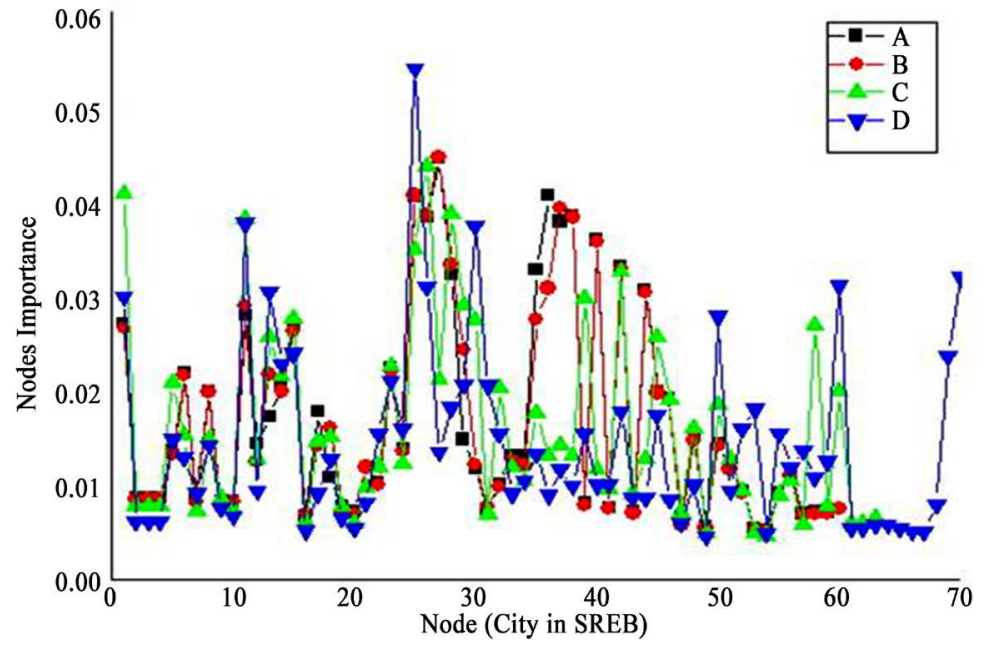

Figure 4. Nodes importance of RNSREB with $\alpha=0.2, \beta=0.4, \gamma=0.4$.

with larger scale will appear in these cities like Geermu, Ruoqiang, Kuerle, Maduo, Panzhihua, etc., meaning that we should pay more attention to those nodes, design and layout related infrastructure in advance. We also find that Chongqing-Chengdu-Geermu-Ruoqiang-Kuerle will be a new rapid channel connecting northwest and southwest China. As the ranking of Xining, Geermu and Maduo is increasingly clear and the status of strategic channel of Qinghai in SREB is also increasingly prominent, Xining, Geermu and Maduo will be the cores of RNSREB and play a critical role.

Studying the RNSREB (outside China), highway network of SREB and airway network of SREB, and considering the traffic, economical, and other factors of these networks will be the future work.

\section{References}

[1] Hu, A.G., Ma, W. and Yan, Y.L. (2014) Connotation, Definition and Passage of "Silk-Road Economic Belt" Strategy. Journal of Xinjiang Normal University (Philosophy and Social Sciences), 2, 1-10.

[2] Harbin Maps Press (2015) Communication Atlas of China. Harbin Maps Press, Harbin.

[3] National Railway Administration (2016) Planning and Developing. http://www.nra.gov.cn/fwyd/hyjc/ghfz

[4] Wang, J.E. and Mo, H.H. (2014) Complex Evolution Process of China’s Air Transport Network. Journal of Transportation Systems Engineering and Information Technology, 1, 71-80.

[5] Wu, J.J., Gao, Z.Y. and Sun, H.J. (2006) Complexity and Efficiency of Beijing Transit Network. International Journal of Modern Physics B, 20, 2119. http://dx.doi.org/10.1142/S0217979206034571

[6] Wang, W. (2011) Analysis and Study on the Survivability of Railway Network. Ph.D. Thesis, Beijing Jiaotong University, Beijing.

[7] Chu, Y.J., Cheng, W.L., Luo, X. and Huang, Z.C. (2010) Empirical Analysis for Attack Tolerance of Bus Network. Journal of Computer Engineering and Applications, 26, 203-205.

[8] Liu, J.G., Ren, Z.M. and Guo, Q. (2013) Node Importance Ranking of Complex Networks. Acta Physica Sinica, 62, Article ID: 178901.

[9] Yu, H., Liu, Z. and Li, Y.J. (2013) Key Nodes in Complex Networks Identified by Multi-Attribute Decision-Making Method. Acta Physica Sinica, 62, Article ID: 020204.

[10] Karygiannis, A., Antonakakis, E. and Apostolopoulos, A. (2006) Detecting Critical Nodes for MANET Intrusion Detection Systems. Second International Workshop on Security, Privacy and Trust in Pervasive and Ubiquitous Computing (SecPerU'06), Lyon, 29-29 June 2006, 9-15. http://dx.doi.org/10.1109/secperu.2006.8

[11] Rao, Y.P., Lin, J.Y. and Zhou, D.F. (2009) Method for Network Invulnerability and Node Importance Evaluation. Computer Engineering, 6, 14-16.

[12] He, N., Li, D.Y., Gan, W.Y. and Zhu, X. (2007) Mining Vital Nodes in Complex Networks. Journal of Computer Science, 12, 1-5. 
[13] Chen, Y., Hu, A.Q. and Hu, X. (2004) Evaluation Method for Node Importance in Communication Networks. Journal of China Institute of Communications, 8, 129-134. http://dx.doi.org/10.1080/02533839.1985.9676814

[14] Huang, J.H. and Huang, F.F. (2013) 2D Index Study on Nodes Importance Evaluation of Complex Networks. Journal of Xidian University (Social Science), 5, 36-42.

Submit or recommend next manuscript to SCIRP and we will provide best service for you:

Accepting pre-submission inquiries through Email, Facebook, LinkedIn, Twitter, etc.

A wide selection of journals (inclusive of 9 subjects, more than 200 journals)

Providing 24-hour high-quality service

User-friendly online submission system

Fair and swift peer-review system

Efficient typesetting and proofreading procedure

Display of the result of downloads and visits, as well as the number of cited articles

Maximum dissemination of your research work

Submit your manuscript at: http://papersubmission.scirp.org/ 